\title{
Review Article \\ Predictive Biomarkers in Colorectal Cancer: From the Single Therapeutic Target to a Plethora of Options
}

\author{
Daniela Rodrigues, ${ }^{1,2}$ Adhemar Longatto-Filho, ${ }^{1,2,3,4}$ and Sandra F. Martins ${ }^{1,2,5}$ \\ ${ }^{1}$ Life and Health Sciences Research Institute (ICVS), School of Health Sciences, University of Minho, Campus of Gualtar, \\ 4710-057 Braga, Portugal \\ ${ }^{2}$ ICVS/3B's-PT Government Associate Laboratory, Braga/Guimarães, Campus of Gualtar, 4710-057 Braga, Portugal \\ ${ }^{3}$ Laboratory of Medical Investigation (LIM) 14, Faculty of Medicine, University of São Paulo, 01246-903 São Paulo, SP, Brazil \\ ${ }^{4}$ Molecular Oncology Research Centre, 14784-400 Barretos, SP, Brazil \\ ${ }^{5}$ Surgery Department, Hospitalar Center Trás-os-Montes e Alto Douro, Unidade Hospitalar de Chaves, \\ Avenida Dr. Francisco Sá Carneiro, 5400-279 Chaves, Portugal
}

Correspondence should be addressed to Sandra F. Martins; sandramartins@ecsaude.uminho.pt

Received 7 December 2015; Revised 17 May 2016; Accepted 4 July 2016

Academic Editor: Anthony Antoniou

Copyright (c) 2016 Daniela Rodrigues et al. This is an open access article distributed under the Creative Commons Attribution License, which permits unrestricted use, distribution, and reproduction in any medium, provided the original work is properly cited.

Colorectal cancer (CRC) is one of the most frequent cancers and is a leading cause of cancer death worldwide. Treatments used for CRC may include some combination of surgery, radiation therapy, chemotherapy, and targeted therapy. The current standard drugs used in chemotherapy are 5-fluorouracil and leucovorin in combination with irinotecan and/or oxaliplatin. Most recently, biologic agents have been proven to have therapeutic benefits in metastatic CRC alone or in association with standard chemotherapy. However, patients present different treatment responses, in terms of efficacy and toxicity; therefore, it is important to identify biological markers that can predict the response to therapy and help select patients that would benefit from specific regimens. In this paper, authors review CRC genetic markers that could be useful in predicting the sensitivity/resistance to chemotherapy.

\section{Introduction}

Colorectal cancer (CRC) is one of the most frequent cancers worldwide, being the third most frequent in men $(10 \%$ of the total) and the second in women $(9.2 \%$ of the total). Moreover, the mortality rate caused by CRC is the fourth highest in men ( $8 \%$ of the total) and the third in women ( $9 \%$ of the total) [1].

Several molecular mechanisms have been identified in CRC carcinogenesis, such as oncogenes activation, tumor suppressor genes inactivation [2], mutations in mismatch repair (MMR) genes, microsatellite instability (MSI) [3], and epigenetic alterations [4]. The accumulation of such alterations ultimately leads to neoplastic transformation.

The standard drugs for CRC chemotherapy are 5-fluorouracil (5-FU) and leucovorin (LV) in combination with irinotecan and/or oxaliplatin [5]. Current guidelines suggest the use of FOLFOX (5-FU/LV with oxaliplatin) or CapeOx (capecitabine and oxaliplatin) in stage III CRC, after surgical resection [6]. The MOSAIC (Multicenter International Study of Oxaliplatin/5-FU/LV in the Adjuvant Treatment of Colon Cancer) trial showed significant improvements in diseasefree survival (DFS) and overall survival (OS) for FOLFOX compared with FL (5-year DFS: 73.3\% versus 67.4\%, resp., $(p=0.003)$ and 6 -year OS $78.5 \%$ versus $76.0 \%(p=0.046))$ in stage III CRC but not in stage II [7]. The use of CapeOx regimen in the treatment of patients with stage III CRC showed an increase in 7-year OS when compared with those treated with 5-FU/LV (73\% versus 67\%; hazard ratio [HR]: 0.83 ; $95 \%$ confidence interval [CI]: $0.70-0.99 ; p=0.04$ ) [8]. In 2009, the PETACC-3 study investigated the efficacy of FOLFIRI (FU/LV with irinotecan) versus the 5-FU/LV regimen in patients with stage III CRC, with no significant differences in 5-year OS (73.6\% versus 71.3\% [ $p=\mathrm{NS}])$ and DFS ( $56.7 \%$ versus $54.3 \%[p=N S])$. Therefore, regimens including irinotecan are not recommended in adjuvant therapy of stage III disease [9]. 
The use of adjuvant chemotherapy has been proven to increase OS in patients with stage III CRC but in stage II CRC this data remain controversial. That may suggest that the use of adjuvant chemotherapy has a greater advantage in people with higher risk [10]. Therefore, National Comprehensive Cancer Network (NCCN) guidelines recommend that treatment with 5-FU/LV, capecitabine, FOLFOX, CapeOx, or bolus 5-FU/LV/oxaliplatin (FLOX) should be considered in patients with stage II CRC and high risk of recurrence, defined as those with T4 tumors (stage IIB/IIC); poorly differentiated histology (exclusive of those cancers that are MSIhigh [MSI-H]); lymphovascular invasion; bowel obstruction; lesions with localized perforation or close, indeterminate, or positive margins; or inadequately sampled nodes $(<12$ lymph nodes). In this setting, analyzing MSI plays a crucial role in the decision of whether to use adjuvant therapy in patients with stage II CRC [6]. This issue will be addressed later in this paper.

Metastatic CRC (mCRC) has been shown to benefit from neoadjuvant and adjuvant chemotherapy. A 2012 metaanalysis combining data from 3 studies and 642 patients showed an increase in DFS (pooled HR, 0.71; CI, 0.582-0.878; $p=0.001$ ) and progression-free survival (PFS) (pooled HR, 0.75; CI, 0.620-0.910; $p=0.003$ ) in CRC patients with liver metastasis treated with surgery plus chemotherapy, when compared to those treated with surgery alone. However, no increase on OS was observed (pooled HR, 0.743; CI, 0.5271.045; $p=0.088$ ) [11]. More recently, another meta-analysis showed similar results regarding perioperative chemotherapy in patients with resectable colorectal hepatic metastasis when compared to surgery alone. The data from 1896 patients from 10 studies showed a significant benefit in DFS in those who received perioperative therapy $(\mathrm{HR}, 0.81$; 95\% CI, 0.72-0.91; $p=0.0007)$ but no significant statistical difference in OS (HR, 0.88; 95\% CI, 0.77-1.01; $p=0.07$ ) [12].

According to current NCCN guidelines, one of the following regimens should be used in systemic chemotherapy of mCRC: FOLFOX, FOLFIRI, CapeOx, infusional 5-FU/LV or capecitabine, or FOLFOXIRI (FU/LV with oxaliplatin and irinotecan) [6]. Over the years, several studies were performed in order to evaluate the efficacy of one regimen over the others. In 2005, the GOIM (Gruppo Oncologico dell'Italia Meridionale) trial compared the efficacy of FOLFIRI versus FOLFOX regimens in 360 patients. The results showed no differences in OS (15 versus 14 months $[p=\mathrm{NS}]$ ), response rate $(\mathrm{RR})$ (31\% versus $34 \%$ [ $p=\mathrm{NS}]$ ), and time to progression (TTP) (7 versus 7 months $[p=\mathrm{NS}]$ ) [13]. Two other clinical trials tried to compare the efficacy and toxicity of FOLFIRI versus FOLFOXIRI regimens. Souglakos et al. found no differences in OS (19.5 versus 21.5 months [ $p=\mathrm{NS}$ ]), RR (34\% versus $43 \%$ [ $p=\mathrm{NS}]$ ), and TTP (6.9 versus 8.4 months [ $p$ $=\mathrm{NS}]$ ) [14]. On the other hand, Falcone et al. showed an improvement in RR (60\% versus 34\% [ $p<0.0001])$ and PFS (9.8 versus 6.9 months $[p=0.0006])$ for the FOLFOXIRI group however at cost of increased toxicity $(p<0.001)$ [15]. A randomized trial compared the efficacy of CapeOx versus FOLFOX, with results showing no significant differences in PFS (8.0 versus 8.5 months, resp.) (HR, 1.04; 97.5\% CI,
0.93-1.16) and OS (19.6 versus 19.8 months, resp.) (HR, 0.99; 97.5\% CI, 0.88-1.12) [16].

Most recently, biologic agents such as cetuximab/panitumumab (monoclonal antibodies directed against the EGFR [epidermal growth factor receptor]) and bevacizumab (a humanized monoclonal antibody that targets VEGF [vascular endothelial growth factor]) have been proven to have therapeutic benefits in $\mathrm{mCRC}$ alone or in association with standard chemotherapy. In the CRYSTAL trial, Van Cutsem et al. investigated the efficacy of cetuximab plus FOLFIRI as first-line treatment for $\mathrm{mCRC}$ versus FOLFIRI alone in a total of 599 patients. There was an increase in PFS in the cetuximab-FOLFIRI group ( $\mathrm{HR}, 0.85$; 95\% CI, 0.72-0.99; $p=$ 0.048 ), but there was no difference in OS ( $\mathrm{HR}, 0.93 ; 95 \% \mathrm{CI}$, $0.81-1.07 ; p=0.31$ ) [17]. Another study involving 813 patients with previously untreated mCRC compared the efficacy of bevacizumab along with irinotecan, bolus fluorouracil, and LV (IFL) versus IFL alone. Results showed an improvement in median survival (HR, $0.66[p<0.001])$, PFS (HR, 0.54; $p<0.001)$, overall response rate $(44.8 \%$ versus $34.8 \%$ [ $p=$ $0.004])$, and median duration of relapse (HR, $0.62 ; p=0.001)$ in the group treated with bevacizumab [18]. Therefore, the use of these targeted agents can be considered as initial therapy for $\mathrm{mCRC}$, together with one of the regimens mentioned above [6].

Despite all the evidence and current recommendations, patients present different responses to treatment, regarding efficacy and toxicity. Therefore, it is important to identify biological markers that can predict the response to therapy and help select patients that would benefit from specific regimens [19].

In this paper, authors review CRC genetic markers that could be useful in predicting the sensitivity/resistance to chemotherapy.

\section{5-Fluorouracil}

5-FU is the basis of CRC chemotherapy regimens. Its antitumor effect relies on the inhibition of thymidylate synthase (TS), the rate-limiting enzyme in the formation of thymidylate (TMP), which is essential for DNA synthesis. 5-FU enters cells and is converted to fluorodeoxyuridine monophosphate, which is the substrate to TS [20].

Studies have shown that tumors that express high levels of TS are associated with resistance to 5-FU [21, 22]. A study correlated the TS protein and gene expression with response to 5-FU based therapy in patients with CRC and gastric cancer. Patients with responsive disease had a mean TS protein level of $0.17 \pm 0.03$ arbitrary units (range, 0.05 to 0.38 ), whereas in patients whose tumors did not respond, the mean TS protein level was significantly higher, $0.60 \pm$ 0.09 (range, 0.06 to $1.01 ; p<0.01$ ). Similarly, in patients with responsive disease, the mean TS : $\beta$-actin gene ratio was $1.36 \pm 0.3$ (range, $0.5-3.3$ ). On the other hand, biopsies from patients with unresponsive disease had a mean TS: $\beta$-actin gene ratio of $15.4 \pm 2.6$ (range, 2.7-35.9; $p<0.01$ ) [21]. A meta-analysis involving twenty studies tried to estimate the prognostic significance of TS expression in both advanced and localized CRC. The combined HR estimate for OS was 
1.74 (95\% CI, 1.34 to 2.26 ) and 1.35 (95\% CI, 1.07 to 1.80 ) in the advanced and adjuvant settings, respectively. However, the authors assume possible existence of heterogeneity and publication bias [22].

The variability in TS expression seems to be related to polymorphisms in the TS promoter enhancer region (TSER), which determines TS expression. The two most common polymorphisms are the $3 \mathrm{R}$ and $2 \mathrm{R}$ variants. One study showed that $\mathrm{mCRC}$ with the $3 \mathrm{R}$ homozygous polymorphism $(3 \mathrm{R} / 3 \mathrm{R})$ had higher levels of TS when compared with the $2 \mathrm{R}$ homozygous variant $(2 \mathrm{R} / 2 \mathrm{R})$. In this trial, 50 patients with $\mathrm{mCRC}$ were treated with 5-FU. Individuals homozygous for the 3R variant had 3.6 times higher TS mRNA levels compared to those homozygous for the $2 \mathrm{R}$ variant in tumor tissue $(p=0.004)$. The authors then evaluated whether the TS polymorphism could predict the clinical outcome. The $\mathrm{RR}$ of individuals carrying the $2 \mathrm{R} / 2 \mathrm{R}$ genotype was $50 \%$ against $9 \%$ of the $3 R / 3 R$ patients $(p=0.041)$. Heterozygous individuals showed RR of $15 \%$. Moreover, patients displaying the $3 \mathrm{R}$ homozygous polymorphism had less severe side effects to 5 -FU $(p=0.008)$ [23].

However, there are $3 \mathrm{R} / 3 \mathrm{R}$ variants that express low levels of TS. The cause appears to be a single nucleotide polymorphism (SNP) in the $3 \mathrm{R}$ allele, in which a guanine is substituted by a cytosine, resulting in low TS expression [24]. Tumors expressing this polymorphism could therefore benefit from therapy with 5-FU.

Another aspect that should be taken into account is the loss of heterozygosity $(\mathrm{LOH})$ that has been observed in the TS locus of CRC. When $\mathrm{LOH}$ is present, in $2 \mathrm{R} / 3 \mathrm{R}$ variant, it could originate either a $2 \mathrm{R} / \mathrm{loss}$ or a $3 \mathrm{R} /$ loss tumor. This would ultimately affect its sensitivity to fluoropyrimidinebased regimens [25].

Thymidine phosphorylase (TP) is another enzyme involved in nucleotide metabolism. Concerning fluoropyrimidine therapy, TP is responsible for the conversion of $5^{\prime}$ deoxy-5-fluorouridine ( $5^{\prime}$-FUR) into 5 -FU. TP has been proven to have a dual role in CRC: it is necessary for 5-FU prodrug activation and it has a role in promoting angiogenesis. Tsujitani et al. investigated the prognostic significance of microvessel density and the relationship between the expression of VEGF, TP, and angiogenesis in patients with gastric carcinoma. Their results revealed that tumors positive for VEGF and TP had high microvessel density, while VEGF and TP negative tumors showed low microvessel density. This evidence strongly suggests an association of TP with tumor neovascularization [26].

Even though high tumor TP expression leads to increased angiogenesis and therefore a poor prognosis (which is linked to increased infiltration, growth, and metastization) [27], it has been documented that it also increases sensitivity to 5FU and thus its effectiveness in CRC treatment [28].

Tumor response to 5-FU therapy also depends on its bioavailability to ultimately inhibit DNA synthesis. DPD is the rate-limiting enzyme in 5-FU catabolism [29]; hence, high levels of DPD are linked with reduced fluoropyrimidine sensitivity, probably due to its decreased cytotoxic effect. Kornmann et al. showed that, among CRC patients treated with 5-FU, those who displayed low levels of DPD survived longer than those with high DPD tumors. They then concluded that DPD may have a prognostic value in CRC patients and those who would benefit the most from 5-FU based chemotherapy would be the ones who express the lowest levels of DPD [30]. Similarly, another study revealed that tumors from CRC patients who responded to therapy with 5-FU presented low levels of DPD [31].

Kornmann et al. tried to determine the prognostic value of TS and dihydropyrimidine dehydrogenase (DPD) in CRC patients receiving 5 -FU. Resulting data showed that, among those treated with this drug, the ones who displayed higher levels of TS presented a better prognosis. Additionally, in each TS group, patients with low DPD survived longer than the ones with high DPD levels. Furthermore, the authors consider that the combination of TP and DPD is an independent prognostic factor for survival $(p=0.030)$ [30].

Nevertheless, individuals with DPD deficiency experience severe toxicity to 5-FU [32, 33]. Van Kuilenburg et al. performed a study, in which they evaluated the importance of DPD deficiency in 5-FU toxicity. They conducted a DPD activity, DPD gene, and clinical presentation analysis after 5FU administration. Results demonstrated that $55 \%$ of patients with a decreased DPD activity suffered from grade IV neutropenia compared with $13 \%$ of patients with a normal DPD activity $(p=0.01)$. Moreover, toxicity was observed twice as fast in patients with low DPD activity as compared with patients with a normal DPD activity (10.0 \pm 7.6 versus $19.1 \pm 15.3$ days; $p<0.05)$ [33].

\section{Irinotecan}

Irinotecan is an inhibitor of DNA topoisomerase I used in CRC treatment, usually in combination with 5-FU, as previously mentioned. It is a prodrug which is then converted to SN-38, the active metabolite that exerts both the antitumor and toxic effects [34]. SN-38 is then conjugated by UDP-glucuronosyltransferase 1A1 (UGT1A1) to SN-38G, an inactive metabolite [35]. UGT1A1 deficiency, caused by some polymorphisms, results in SN-38 accumulation, causing irinotecan-related toxicity, which includes diarrhea, dehydration and neutropenia.

The UGT1A1 enzyme is also involved in converting bilirubin into more soluble forms. Therefore, UGT1A1 deficiency can also lead to accumulation of unconjugated bilirubin, as seen in Crigler-Najjar and Gilbert syndromes [36, 37].

The UGT1A1* 28 polymorphism has been linked to severe side effects, namely, neutropenia and diarrhea. In this variant, the UGT1A1 expression is markedly decreased which leads to prolonged exposure to SN-38 [38]. In a 2014 meta-analysis, sixteen studies were included in order to investigate the association of UGT1A1 polymorphisms and irinotecan-induced diarrhea and neutropenia. UGT1A1* $28 /{ }^{*} 28$ genotype was associated with more than fourfold (OR, 4.79; 95\% CI, 3.287.01; $p<0.00001)$ and threefold (OR, 3.44; 95\% CI, 2.454.82; $p<0.00001$ ) increases in the risk of neutropenia when compared with wild type and with at least one UGT1A1*1 allele, respectively. Moreover, $\mathrm{UGT} 1 \mathrm{A1}{ }^{*} 28 /{ }^{*} 28$ genotype was associated with a twofold risk of diarrhea (OR, 1.84; 95\% CI, $1.24-2.72 ; p=0.002)$. Additionally, the incidence of diarrhea 
was higher when irinotecan was given at higher doses (OR, 2.37; 95\% CI, 1.39-4.04; $p=0.002$ ) [37]. In 2005, the Food and Drug Administration (FDA) recommended that individuals homozygous for UGT1A1* 28 polymorphism would start the treatment with irinotecan with lower doses.

While UGT1A1*28 is a common variant in both Caucasians and Asians, $\mathrm{UGT1A1}^{*} 6$ is another polymorphism that is only found in Asians and has similar toxic effects of those of the UGT1A1 ${ }^{*} 28$ variant. A systematic review analyzed the association between $\mathrm{UGT1A1}^{*} 6$ polymorphisms and irinotecan-induced neutropenia and diarrhea in Asian patients. Patients with $\mathrm{UGT}_{1 \mathrm{~A}}{ }^{*} 6 /^{*} 6$ genotype displayed an increased risk for severe neutropenia (OR, 4.44; $95 \% \mathrm{CI}$; $2.42-8.14 ; p<0.001)$. Individuals carrying the heterozygous variant also showed a higher risk for neutropenia, although not as high as the homozygous variant (OR, 1.98; 95\% CI; $1.45-2.71 ; p<0.001)$. UGT1A1 ${ }^{*} 6$ homozygous patients were at higher risk for severe diarrhea (OR, 3.51; 95\% CI; 1.418.73; $p=0.007$ ), while heterozygous patients had no significant risk. Therefore, UGT1A1* 6 polymorphisms are potential biomarkers predicting irinotecan-induced severe toxicity in Asian patients. In this context, since 2008, Japan has been recommending the screening of patients for these two polymorphisms [39].

Even though genotyping for the UGT1A1 polymorphisms could be important in order to prevent severe adverse effects such as neutropenia, it does not predict response to chemotherapy.

\section{Oxaliplatin}

Oxaliplatin is a platinum analog chemotherapeutic agent with a 1,2-diaminocyclohexane (DACH) carrier ligand. It forms adducts on DNA strands, leading to DNA damage [26]. The nucleotide excision repair (NER) pathway is responsible for the removal of these adducts, reducing sensitivity to oxaliplatin. The two major genes involved in the NER pathway are excision repair cross-complementation group 1 (ERCC1) and xeroderma pigmentosa [27]. ERCC1 overexpression has been associated with resistance to oxaliplatin, probably because it induces repair of DNA strands. In 2001, Shirota et al. revealed that intratumoral ERCC1 mRNA expression levels are an independent predictive marker of survival for 5-FU and oxaliplatin combination chemotherapy in 5-FU-resistant mCRC. Patients with a low ERCC1 expression level had a greater median survival time than those with high levels of ERCC1 mRNA (10.2 versus 1.9 months, resp.; $p<0.001$ ) [40]. A more recent study involving 180 stage III CRC patients treated with FOLFOX-4 showed that ERCC1 overexpression is an important predictor of early failure $(p=0.005)$, DFS $(p<0.001)$, and OS $(p<0.001)$ [41]. However, conflicting data have been observed about the association of ERCC1 and clinical outcome in CRC patients treated with oxaliplatin. Li et al. conducted a trial to investigate the relationship between ERCC1 and DFS in Chinese CRC patients receiving oxaliplatin plus 5-FU. Results failed to show ERCC1 mRNA levels as a predictive factor for DFS $(p=0.638)$ [42]. Similar conclusions were achieved by Kim et al. in stage II and stage III CRC patients who underwent a curative resection followed by FOLFOX-4 adjuvant chemotherapy. ERCC1 expression was not significantly correlated with the 5 -year DFS ( $p=0.396)$ and therefore cannot be used as a prognostic factor [43].

Based on the inconsistent results concerning ERCC1 role in predicting patients' response to therapy, a meta-analysis was performed in order to elucidate the prognostic role of ERCC1 expression in patients with CRC. A total of 11 studies were analyzed. Results revealed that patients with higher ERCC1 expression showed a poorer OS (HR, 2.325; 95\% CI; $1.720-3.143$; $p<0.001$ ), PFS (HR, 1.917; 95\% CI; 1.366-2.691; $p<0.001$ ), and response to chemotherapy (HR, 0.491; 95\% CI; 0.243-0.990; $p=0.047$ ), when compared to those with low ERCC1 expression. Therefore, the authors conclude that ERCC1 can indeed be a prognostic factor for OS, PFS, and response to chemotherapy [44]. However, further studies need to be addressed in order to better understand the true prognostic value of this biomarker.

Some studies showed that ERCC1-118 polymorphism influences response to oxaliplatin-based therapy. Furthermore, the allele ERCC1-118T induces higher levels of ERCC1 mRNA, which may explain why it is associated with shorter PFS in patients with $\mathrm{mCRC}$ treated with FOLFOX-4 regimen $[45,46]$. This was also documented for XPD-751 polymorphism holding the $\mathrm{C}$ allele. Moreover, the combination of ERCC1-118 T/T genotype with either XPD-751 A/C or XPD$751 \mathrm{C} / \mathrm{C}$ resulted in an even shorter PFS (HR, 2.84; 95\% CI, $1.47-0.45 ; p=0.002)$, compared to the case when only one of these genotypes was present [46]. More recently, another study showed that the XPG Arg1104His polymorphism variants were associated with a longer DFS in patients receiving oxaliplatin-based chemotherapy [47]. Based on current data, ERCC1 screening is not recommended to select patients for oxaliplatin-based regimens.

\section{Antibodies Targeting EGFR}

The activation of EGFR induces cell proliferation by activating two main downstream signaling pathways, the Ras/Raf/ mitogen-activated protein kinase (MAPK) (mainly responsible for cell proliferation) and the PI3KPTEN-AKT (mainly responsible for cell survivor) pathways.

The monoclonal antibodies cetuximab and panitumumab are directed against EGFR, therefore inhibiting cell proliferation and tumor growth, by binding to the extracellular domain of EGFR and blocking ligand-induced receptor phosphorylation and further signaling [48]. Response to EGFRtargeted therapy varies greatly between individuals, and it is not correlated with EGFR levels. A clinical trial conducted by Cunningham et al. showed that the degree of EGFR expression, either as the percentage of EGFR-positive tumor cells or as the maximal staining intensity per cell, had no relation to the clinical response rate $(p=0.67$ and $p=0.84$, resp.) [49]. Another study, designed to access the antitumor activity and toxicity of cetuximab in patients whose tumors express the EGFR, displayed similar results. Moreover, $88 \%$ of the patients presented skin reactions, such as acne-like rash and paronychia cracking, as well as allergic reactions, which in two cases led to therapy cessation. The authors also verified 
a statistical correlation between the presence and severity of the acne-like rash and survival ( $p=0.02)$ [50]. Given the possible adverse effects of cetuximab, it is important to be able to select the patients that would benefit from therapy with this agent. Once scientific evidence showed no correlation between EGFR expression and clinical response, it was hypothesized that gene mutations in downstream proteins could be responsible for cetuximab/panitumumab resistance.

KRAS has been widely studied in the context of cetuximab/panitumumab response in mCRC. Several studies showed that KRAS mutations are highly predictive of cetuximab resistance in patients with mCRC. The most common KRAS mutations occur at exon-2 (codons 12 and 13), although those have also been found in exon-3 and exon-4 [51-53].

As previously mentioned, the CRYSTAL trial was conducted to investigate the efficacy of cetuximab plus irinotecan, 5-FU, and LV (FOLFIRI) as first-line treatment for mCRC [17]. The results showed improvement in PFS but not in OS in patients treated with cetuximab. However, in a subset analysis of patients with wild-type KRAS, the addition of cetuximab to FOLFIRI resulted in significant improvements in OS (median, 23.5 versus 20.0 months; $\mathrm{HR}=0.796 ; p=$ 0.0093), PFS (median, 9.9 versus 8.4 months; $\mathrm{HR}=0.696$; $p=0.0012$ ), and $\mathrm{RR}(57.3 \%$ versus $39.7 \%$; $\mathrm{OR}=2.069 ; p=$ 0.001 ) compared with FOLFIRI alone. No significant difference in efficacy was evident in patients with mutant KRAS (codons 12 and 13, exon-2) [17, 54]. Most recently, a revision of the CRYSTAL study showed that not only the mutations in codons 12 or 13 were linked to worst response to cetuximab plus FOLFIRI, but also the same was true to mutations in exon-3 and exon-4. DNA samples from CRYSTAL patients that presented a KRAS wild-type status for mutations in codons 12 and 13 were reanalyzed for mutations in four additional KRAS codons (exon-3 and exon-4) and six NRAS codons (exon-2, exon-3, and exon-4). Results showed no differences in OS and PFS between patients treated with cetuximab plus FOLFIRI and FOLFIRI alone [55].

Three important studies were conducted in order to assess the efficacy of FOLFOX plus cetuximab versus FOLFOX alone. In the OPUS (Oxaliplatin and Cetuximab in FirstLine Treatment of Metastatic Colorectal Cancer) study, Bokemeyer et al. showed that the addition of cetuximab to FOLFOX-4, as first-line therapy in patients with mCRC, resulted in better response to chemotherapy $(\mathrm{OR}=2.551$; $p=0.0027)$ and PFS (HR $=0.567 ; p=0.0064)$ in wild-type KRAS individuals when compared with those treated with FOLFOX-4 alone. Those who held the KRAS exon-2 mutations showed no benefit from cetuximab, as they presented with worse response to treatment and shorter PFS [56]. Another study called COIN also compared the efficacy of FOLFOX plus cetuximab versus FOLFOX alone in 1630 patients. In this trial, Maughan et al. showed that FOLFOX with cetuximab increased RR compared with FOLFOX alone ( $64 \%$ versus $57 \%$, resp.; $p=0.049$ ). However, no evidence of improved PFS (8.6 versus 8.6 months, resp.; $\mathrm{HR}=0.96,95 \%$ $\mathrm{CI}=0.82-1.12$, and $p=0.60$ ) or OS (17.0 versus 17.9 months; $\mathrm{HR}=1.04,95 \% \mathrm{CI}=0.87-1.23$, and $p=0.67)$ was seen among patients with wild-type KRAS. Furthermore, skin and gastrointestinal toxic effects were more frequent in the group treated with FOLFOX plus cetuximab compared to control group (14 versus 114 and 67 versus 97, resp.) in patients with KRAS wild-type tumors [57].

A multicenter phase III trial named NORDIC-VII investigated the efficacy of cetuximab plus a bolus of fluorouracil/folinic acid and oxaliplatin (Nordic FLOX), administered continuously or intermittently, in 571 patients with previously untreated mCRC. Therefore, the patients were randomly divided into three groups, one receiving standard Nord FLOX ( $\operatorname{arm} \mathrm{A}$ ), another receiving FLOX plus cetuximab ( $\operatorname{arm} B)$, and a third group treated with cetuximab plus intermittent FLOX (arm C). OS, PFS, and RR were similar in the 3 treatment arms (OS $=20.4,19.7$, and 20.3 months, resp. $[p=\mathrm{NS}]$; PFS $=7.9,8.3$, and 7.3 months $[p=\mathrm{NS}]$; and RR: $41 \%, 49 \%$, and $47 \%$ [ $p=N S]$ ). In KRAS wild-type tumors, the addition of cetuximab did not bring about additional benefits. In KRAS mutant tumors, improvement in PFS was reported in $\operatorname{arm} B$ for patients treated with cetuximab when compared with those receiving FLOX alone. However, this difference was not statistically significant (7.8 versus 9.2 months; HR = $0.71 ; p=0.07$ ) [58]. Based on these studies, the screening of patients with mCRC to exon-2 KRAS mutations is currently recommended. However, $65 \%$ of the wild-type patients for the exon-2 mutation are resistant to cetuximab, which brings up the importance of using new biomarkers such as exon-3 and exon-4 mutations [59].

KRAS has also been studied in the setting of panitumumab use in patients with chemotherapy-refractory mCRC. Also in this case, only the KRAS wild-type patients showed sensitivity to monotherapy with panitumumab. Amado et al. conducted a phase III trial, in which they compared the efficacy of panitumumab as monotherapy for mCRC to best supportive care (BSC). Additionally, the authors of this study tried to evaluate whether the efficacy of panitumumab was related to KRAS status. Results showed that, in those treated with panitumumab, PFS was significantly increased (HR, 0.45; 95\% CI, 0.34-0.59; $p<0.0001$ ) in the wild-type KRAS group compared to KRAS mutant group (HR, 0.99; 95\% CI, 0.73-1.36). In patients with KRAS wild-type mCRC tumors, treatment with panitumumab resulted in higher PFS (HR, 0.45; 95\% CI, 0.34-0.59; median PFS of 12.3 weeks for panitumumab versus 7.3 weeks for BSC). Contrarily, in the KRAS mutant group, no improvement in PFS was observed with therapy with panitumumab (HR, 0.99; 95\% CI, 0.73-1.36; median PFS of 7.4 weeks for panitumumab versus 7.3 weeks for BSC) [60]. The phase III PRIME trial compared the efficacy and safety of FOLFOX-4 versus FOLFOX-4 plus panitumumab in mCRC patients with no previous chemotherapy treatment. Data from 1183 patients was collected. Among patients with KRAS wild-type tumors, results displayed a significant improvement in PFS in the group treated with FOLFOX-4 and panitumumab, when compared to the FOLFOX-4 group (9.6 versus 8.0 months, resp.; HR, 0.80; $95 \%$ CI, $0.66-0.97 ; p=0.02)$. No differences in OS were observed (23.9 versus 19.7 months, resp.; HR, 0.83; 95\% CI, 0.67-1.02; $p=0.072$ ). On the other hand, in the mutant KRAS stratum, there was a significant decrease in PFS in the FOLFOX-4 plus panitumumab group (HR, 1.29; 95\% CI, $1.04-1.62 ; p=0.02$ ). Median OS was 15.5 months versus 19.3 
months, respectively (HR, 1.24; 95\% CI, 0.98-1.57; $p=0.068)$ [61]. Therefore, patients who are candidates to therapy with panitumumab are indicated for screening for KRAS codons 12 and 13 mutations [60].

BRAF is an oncogene that encodes a downstream effector of KRAS in the MAPK pathway. Mutations in this gene may explain some of the cases in which KRAS wild-type patients do not respond to cetuximab/panitumumab therapy. The most common mutation found on BRAF is V600E, which has been shown to induce resistance to EGFR-targeted therapy. In a retrospective study, Di Nicolantonio et al. analyzed tumor responses, PFS, OS, and the mutational status of KRAS and BRAF in 113 tumors from cetuximab- or panitumumabtreated mCRC patients [62]. Data showed decreased PFS $(p=0.011)$ and OS $(p<0.0001)$ in patients with BRAF mutations. Furthermore, none of the patients that displayed the BRAF mutation responded to therapy and none of the responders carried this mutation $(p=0.029)$. This evidence suggested that the introduction of the V600E allele impaired the therapeutic effect of cetuximab/panitumumab. Therefore, the authors hypothesized that a BRAF inhibitor could restore the sensitivity to these drugs. Sorafenib, a clinically approved small-molecule kinase inhibitor, was then used to pharmacologically target BRAF. Results showed that sorafenib restored sensitivity to panitumumab or cetuximab of CRC cells carrying the V600E allele [62]. Additionally, KRAS and BRAF mutations have been shown to be mutually exclusive, with none of the KRAS-mutated samples carrying concomitant BRAF mutations, or vice versa. This evidence had been previously displayed in other studies [62-64].

Another retrospective study evaluated the effect of KRAS downstream mutations on the efficacy of cetuximab in 1022 patients with chemotherapy-refractory mCRC treated with cetuximab plus chemotherapy. Regarding the BRAF status, BRAF mutants had a significantly lower RR (8.3\% [2/24] versus 38.0 [124/326] for wild types; OR, 0.15; 95\% CI, 0.02$0.51 ; p=0.0012)$ and disease-control rate $(37.5 \%[9 / 24]$ versus 77.3\% [252/326]; OR, 0.176; 0.071-0.41; $p<0.0001)$ and shorter PFS (median, 8 versus 26 weeks in wild types; HR, 3.74; 95\% CI, 2.44-5.75; $p<0.0001$ ) and OS (median, 26 versus 54 weeks in wild types, HR, 3.03; $1.98-4.63 ; p<$ 0.0001 ), when compared to wild types [65]. Although some retrospective studies showed a decreased response to cetuximab/panitumumab in patients with BRAF mutations, some patients may have some benefit from therapy with cetuximab as a front-line therapy $[54,66]$.

Therefore, the role of BRAF mutation in predicting response to EGFR-targeting agents is still unclear. On the other hand, its utility as a prognostic factor is more consensual $[54,67]$. Van Cutsem et al. showed that BRAF V600E mutation was linked to a worse prognosis in KRAS wild-type patients, regardless of which treatment they were submitted to (cetuximab plus FOLFIRI versus FOLFIRI alone) [54]. Additionally, the above-mentioned COIN trial revealed that, irrespective of treatment received, OS was shorter in patients whose tumors had mutations in BRAF ( $n=102,8.8$ months, IQR: 4.5-16.1) than in those with BRAF wild-type tumors. This data confirms once again that BRAF mutation is an indicator of poor prognosis [57].
Despite the still controversial role of this downstream mutation as a predictive marker, the NCCN panel recommends the BRAF genotyping for stage IV CRC, at the time of diagnosis. They consider the response to cetuximab/panitumumab in BRAF mutant mCRC to be highly unlikely [6].

PTEN is a member of the EGFR signaling pathway, PI3KPTEN-AKT. In 2007, Frattini et al. showed that loss of PTEN expression was predictive of cetuximab resistance in mCRC patients. In a study involving 27 patients, 11 presented loss of PTEN activity, and none of those responded to therapy with cetuximab $(p<0.001)$. Furthermore, patients who responded to cetuximab presented with PTEN expression and wild-type KRAS genotype [68].

PIK3CA mutations were also linked with resistance to EGFR-targeted therapy $[69,70]$. However, the role of PIEK/ PTEN on predicting response to cetuximab/panitumumab is still controversial. A recent study showed that neither PIK3CA mutations nor PTEN expression levels would predict mCRC patients' response to cetuximab [71].

Besides the molecules that integrate the EGFR signaling pathways, molecules that act in an autocrine or paracrine way may play a role in predicting the response to cetuximab/ panitumumab [72]. Amphiregulin and epiregulin are EGFR ligands whose increased expression has been linked to a good response to EGFR-targeted therapy $[73,74]$.

\section{Bevacizumab}

Bevacizumab is a monoclonal antibody that targets VEGF$A$ and it is used in the treatment of $\mathrm{MCRC}$ in addition to chemotherapy. 18 VEGF-A is a proangiogenic ligand which (together with other proangiogenic molecules) has been associated with tumor vascularization, progression, and metastization. Moreover, it is known to blunt the cytotoxic effect of chemotherapy by recruiting endothelial cells, protecting the tumor from chemotherapy. Bevacizumab blocks this process, by "normalizing" tumor's vascularization and allowing chemotherapy and oxygen to reach tumor cells [75]. Studies have been made in order to identify biomarkers that could predict patients' response to bevacizumab. High VEGF-A levels have been associated with a poor clinical outcome. However, they do not predict patients' response to bevacizumab, as shown by an initial comprehensive analysis of plasma VEGF-A as a biomarker across multiple phase III trials, which included the AVF2107 study [76]. In another study, Hayashi et al. showed that an early increase in VEGF-A circulating levels, after an initial decrease (in patients treated with bevacizumab plus FOLFIRI), was associated with a reduced PFS ( $p=0.009)$ [77].

A 2013 study evaluated the predictive and prognostic value of VEGF using samples from HORIZON II and III trials, in which CRC patients were treated with cediranib, a VEGF receptor (VEGFR) tyrosine kinase inhibitor. The resulting data was consistent with previous studies, with high levels of VEGF associated with worse prognosis, independent of treatment received (HORIZON II OS: HR, 1.35; 95\% CI, 1.12-1.63; HORIZON III OS: HR, 1.32; 95\% CI, 1.12-1.54). However, baseline VEGF was not predictive of response to cediranib [78]. 
Although several studies linked high VEGF levels to a poor clinical outcome, most failed to demonstrate its value as a predictive biomarker for chemotherapy response. Given the importance of finding markers that would help select candidates for treatment with bevacizumab, Bruhn et al. conducted a trial, in which they determined tumor expression levels of the proangiogenic proteins (IL-6, IL-8, b-FGF, PDGF-BB, and VEGF-A) in mCRC patients. Individuals were divided into two groups (high or low), according to their levels of protein expression. Final data showed that low VEGF-A levels were linked to a better RR for bevacizumab (RR [low] 53\% versus [high] 19\%, interaction $p=0.03$ ), while "high" VEGF-A was prognostic for shorter PFS (unadjusted HR: $1.34, p=0.06$; adjusted HR: $1.55, p=0.008)$. The other proangiogenic molecules showed neither a prognostic nor a predictive value [79].

More recently, Tsai et al. performed a clinical trial to compare pre- and posttherapeutic VEGF immunohistochemical (IHC) expression in $57 \mathrm{mCRC}$ patients treated with FOLFIRI plus bevacizumab. Results showed that low posttherapeutic VEGF expression $(p<0.001)$ and decreased peritherapeutic VEGF expression $(p<0.001)$ were significantly predictive factors for therapy response. Moreover, patients with decreased peritherapeutic VEGF expression had improved 6month PFS than those with no peritherapeutic VEGF alterations $(p=0.033)$ [80]. Given the inconsistent data regarding the role of VEGF in response to therapy, its use as a predictive biomarker for treatment with bevacizumab is currently not advised. Besides VEGF-A, several other molecules play a role in angiogenesis, some of which have been the object of clinical studies in order to identify biomarkers of bevacizumab resistance. Although some of them have shown a promising role as predictive markers, there is yet not enough data to support their use in the clinical practice $[77,81-$ 83]. Further investigation is needed in the setting of mCRC patients' sensitivity/resistance to therapy with bevacizumab.

\section{Microsatellite Instability}

Microsatellites are short tandem DNA repeats that occur throughout the genome. When mutations in microsatellites occur in MMR genes (MSH2, MLH1, MSH6, and PMS2), allowing DNA replication errors to occur, they give rise to a defective MMR (dMMR) system. This is usually measured by either the presence of MSI or the absence of the protein products for the MMR genes. MSI tumors can be classified as MSIH or MSI-low (MSI-L), depending on the extent of instability in the marker tested. Tumors that do not present MSI are called microsatellite-stable (MSS). MSI-H occur in about $15 \%$ of CRC (in this case, the hypermethylation of the MLH1 gene promoter inactivates the mismatch repair), particularly in stage II CRC. MSI-H are characterized by a proximal colon predominance, older age, and female sex $[84,85]$.

MSI tumors have been linked to a good prognosis and resistance to fluoropyrimidine-based chemotherapy alone. In 2003, Ribic et al. investigated the usefulness of MSI status as a predictor of the benefit of adjuvant chemotherapy with fluorouracil in stage II and stage III colon cancer. 570 patients from five previous randomized trials who had been randomly assigned to receive adjuvant 5-FU based chemotherapy after surgical resection or no treatment were studied. Results showed that 5-FU based therapy benefited those with MSS or MSI-L tumors but not those who presented a MIS-H phenotype. Patients with MSS who had MSI-L tumors and were submitted to adjuvant therapy showed improved OS (HR, 0.72; 95\% CI, 0.53-0.99; $p=0.04$ ). By contrast, MSI-H tumor patients did not benefit from adjuvant chemotherapy. Patients who did not receive adjuvant therapy and presented MSI-H tumors showed a better five-year rate of OS than patients with tumors exhibiting a MSS or MSI-L status (HR, 0.31; 95\% CI, 0.14-0.72; $p=0.004$ ) [86].

In a similar study, Sargent et al. examined the MMR status as a predictor of adjuvant 5-FU based therapy benefit in patients with stages II and III colon cancer. As in the previous study, a group of 457 patients were randomly assigned to receive either 5-FU based adjuvant therapy or no postsurgical treatment. Patients with MSS tumors who received adjuvant therapy showed a significantly improvement in DFS (HR, 0.67; 95\% CI, 0.48-0.93; $p=0.02$ ). On the other hand, defective MMR (dMMR) tumor patients showed no benefit from 5-FU based therapy when compared with those treated with surgery alone (HR, 1.10; 95\% CI, $0.42-2.91 ; p=0.85)$. In patients with stage II, dMMR CRC, treatment was associated with reduced OS (HR, 2.95; 95\% CI, 1.02-8.54; $p=0.04$ ) [87].

As mentioned above, MSI tumors are associated with a good prognosis. Data from the PETACC-3 study showed that the MSI-H profile occurs more often in stage II CRC than in stage III (22\% versus $12 \%$, resp.; $p<0.0001)$ [88]. Furthermore, evidence showed that these tumors may have a decreased likelihood to metastasize. Koopman et al. collected data from a phase III study in 820 advanced CRC patients, in which dMMR was only found in 18 (3.5\%) patients [85].

All these evidences suggest that stage II CRC with a MSI$\mathrm{H}$ profile may have good prognosis and would not benefit from adjuvant 5-FU based chemotherapy. On the other hand, in stage III disease, the benefit of adjuvant 5-FU based chemotherapy is well documented. Flejou et al. studied the benefit from adding oxaliplatin to a 5 -FU based regimen in stage III CRC, using data from MOSAIC study patients.

This report had previously shown that combining oxaliplatin with 5-FU and LV improves OS and DFS, as mentioned before [7]. After analyzing the MMR status of 986 patients, Flejou and his colleagues displayed data that supports the use of FOLFOX-4 regimen in stage III CRC with a MSI-H profile. HR for stage III dMMR CRC are $0.56(0.19-1.61)$ for RFS, $0.51(0.18-1.41)$ for DFS, and $0.44(0.15-1.34)$ for OS, respectively. HR for stage II dMMR tumors are $0.64(0.11-$ 3.70) for RFS, 0.60 (0.17-2.09) for DFS, and 0.52 (0.13-2.10) for OS, respectively [89].

While stage III CRC should be treated with 5-FU plus oxaliplatin, stage II CRC treatment is more controversial, as these patients do not benefit as much from adjuvant therapy [62]. Patients with stage II disease often display good prognosis and have been shown to be resistant to 5-FU based regimens. Therefore, the NCCN currently recommends determining MSI status for all patients with stage II CRC, and adjuvant treatment should not be given to patients with low-risk MSI-H tumors [6]. 
TABLE 1: Summary of potential predictive biomarkers, available data, and current recommendations.

\begin{tabular}{|c|c|c|c|}
\hline Drug & Potential biomarkers & Data available & Current recommendations \\
\hline \multirow{3}{*}{$5-\mathrm{FU}$} & TS expression & Inconsistent results & None \\
\hline & TP expression & $\begin{array}{l}\text { Inconsistent results; high } \\
\text { level potential indicator of } \\
\text { poor prognosis }\end{array}$ & None \\
\hline & DPD expression & $\begin{array}{l}\text { Not predictive; low levels } \\
\text { associated with better } \\
\text { prognosis }\end{array}$ & None \\
\hline Irinotecan & $\begin{array}{l}\text { UGT1A1 }{ }^{*} \text { 28/UGT1A1 }{ }^{*} 6 \\
\text { polymorphisms }\end{array}$ & $\begin{array}{l}\text { Not predictive; associated } \\
\text { with severe toxicity }\end{array}$ & $\begin{array}{l}\text { Patient screening for } \\
\text { UGT1A1 }{ }^{*} 28 \text { and } \\
\text { UGT1A1 }^{*} 6 \text { (in Japan) } \\
\text { polymorphisms }\end{array}$ \\
\hline Oxaliplatin & ERCC1 & Inconsistent results & None \\
\hline \multirow{4}{*}{ Cetuximab/panitumumab } & KRAS mutation & $\begin{array}{l}\text { Resistance to } \\
\text { cetuximab/panitumumab }\end{array}$ & $\begin{array}{l}\text { Patient screening for } \\
\text { exon-2 KRAS mutations }\end{array}$ \\
\hline & BRAF V600E & $\begin{array}{l}\text { Inconsistent results; poor } \\
\text { prognosis }\end{array}$ & $\begin{array}{l}\text { BRAF genotyping for stage } \\
\text { IV CRC }\end{array}$ \\
\hline & PTEN loss & Not predictive & None \\
\hline & PIK3CA mutation & Not predictive & None \\
\hline \multirow[t]{2}{*}{ Bevacizumab } & VEGF expression & $\begin{array}{l}\text { Not predictive; } \\
\text { contradictory data }\end{array}$ & None \\
\hline & MSI-H & $\begin{array}{l}\text { Resistance to } \\
\text { chemotherapy; good } \\
\text { prognosis }\end{array}$ & $\begin{array}{l}\text { Determination of MSI } \\
\text { status for all stage II CRC } \\
\text { patients }\end{array}$ \\
\hline
\end{tabular}

5-FU: 5-fluorouracil; TS: thymidylate synthase; TP: thymidine phosphorylase; DPD: dihydropyrimidine dehydrogenase; UGT1A1: uridine diphosphate glucuronosyltransferase 1A1; ERCC1: excision repair cross-complementation group 1; VEGF: vascular endothelial growth factor; MSI-H: microsatellite instability-high.

Despite all these studies and NCCN recommendations, a recent meta-analysis concluded that there are no differences in the effect of 5-FU based chemotherapy, regarding the MSI status.

Although there was improvement in DFS (HR, 0.62; 95\% $\mathrm{CI}, 0.54-0.71$ ) and OS (HR, 0.65; 95\% CI, 0.54-0.79) in patients with MSS tumors treated with 5-FU compared with those who were untreated and no improvement in the MSI group (DFS [HR, 0.84; 95\% CI, 0.53-1.32] and OS [HR, 0.66; 95\% CI, 0.43-1.03]), the effect of adjuvant therapy was not different at a statistical significant level [90].

Summary of potential predictive biomarkers, available data, and current recommendations can be observed in Table 1.

\section{Discussion}

CRC is one of the most frequent and deadliest cancers worldwide [1]. Systemic chemotherapy in the adjuvant and advanced setting has evolved greatly in the last decades from basic cytotoxic agents to combination regimens and, more recently, the advent of biological agents [5]. However, patients often present different responses and grades of toxicity to each regimen. Therefore, it is important to identify biomarkers that could predict response to treatment, thus helping us in selecting the best candidates for each drug. In this paper, we reviewed the main molecular markers that have been studied in the setting of CRC.
Studies regarding EGFR-targeting agents cetuximab and panitumumab have so far presented the most consistent results. As discussed before, current guidelines recommend screening patients for KRAS exon-2 mutations who are considered for cetuximab/panitumumab treatment. Wild-type KRAS patients benefit from improvement in PFS, OS, and RR with cetuximab, as shown in CRYSTAL and OPUS trials [17, 56]. In contrast, individuals who presented KRAS mutation had shorter PFS and OS. However, COIN and NORDIC-VII failed to show association between KRAS status and response to therapy with cetuximab $[57,58]$.

In the PRIME trial, evidence showed that wild-type individuals who received panitumumab displayed better PFS, while those with mutant KRAS had no profit from this agent [61].

Therefore, we hypothesized that, in those cases in which KRAS wild-type patients appeared to be resistant to EGFRtargeting agents, other unidentified mutations may be present and may be the cause for the lack of response. Thus, further studies should be addressed in order to investigate the role of KRAS exon-3 and exon-4 mutations as predictive biomarkers for therapy with cetuximab/panitumumab.

BRAF V600E mutation has shown some prognostic value in CRC patients. Nevertheless, its predictive value is still not established, as current studies show inconsistent results. Still, the NCCN recommends the screening for BRAF mutations before initiating treatment with cetuximab/panitumumab [6]. 
Even though current data strongly supports the use of chemotherapeutic agents in stage III and stage IV CRC, the use of adjuvant treatment in stage II disease is still controversial. In this setting, MSI status has been studied as a predictor of the benefit of adjuvant chemotherapy. Tumors displaying MSI-H phenotype have been shown to have better prognosis, as demonstrated in the PETACC-3 study [88]. They also appear to have a lower likelihood to metastasize. Moreover, scientific evidence established a predictive role for the MSI status. In fact, MSI-H tumors seem to have no benefit from 5-FU based chemotherapy $[86,87]$. On the other hand, in stage III CRC patients who were submitted to adjuvant chemotherapy, MSI-H tumors have shown improvement in DFS and OS [89]. So it seems that low-risk stage II CRC, as are the ones that present MSI-H status, should not be submitted to cytotoxic therapy. Conversely, high-risk stage II CRC may have a clinical behavior closer to that of stage III disease and so would benefit from adjuvant chemotherapy. Therefore, the determination of the MSI status in stage II CRC patients is recommended to assess whether they are candidates to receive adjuvant treatment.

Irinotecan has been linked to severe toxicity, particularly in patients with a UGT1A1 deficiency.

The UGT1A1 ${ }^{*} 28$ polymorphism seems to be responsible for low UGT1A1 expression levels and thus a higher frequency and severity of side effects. Moreover, in the Asian population, the $\mathrm{UGT1A1}^{*} 6$ variant was also identified and was associated with severe neutropenia and diarrhea [37]. Hence, recommendations advise the screening of all patients for the UGT1A1 28 and UGT1A1* 6 (in Japan) variants before initiating treatment with irinotecan [39]. Although these polymorphisms can help predict the occurrence of severe toxicity, they are not predictors of therapy response.

So far, none of the other markers have proven a predictive value to therapy response. Studies concerning ERCC1 have shown contradictory results. Regarding the anti-VEGFR agent bevacizumab, data revealed that high VEGF-A levels are associated with poor prognosis.

However, studies addressing the role of VEGF as a predictive biomarker have shown inconsistent results. Thus, VEGF is not currently considered a useful marker of response to bevacizumab. In the future, studies addressing the role of other proangiogenic molecules should be conducted. Several angiogenesis-related factors, such as IL-8, soluble angiopoietin II (sANG-2), basic FGF (b-FGF), and stem cell factor (SCF), have been shown to be increased in CRC patients that did not respond to bevacizumab. However, the available data is still insufficient [77].

\section{Conclusion}

In this paper, the authors reviewed the role of actual predictive biomarkers in CRC. KRAS is the best studied marker of response to cetuximab/panitumumab, with current use in clinical practice. Similarly, patients' genotyping for MMR mutations is also recommended in stage II CRC. Regarding toxicity, individuals who are candidates for therapy with irinotecan should be tested for UGT1A1 polymorphisms.
Besides these, several other molecules have been studied as potential biomarkers for chemotherapy sensitivity/resistance. However, they are not used in practice, as they have not yet proven their clinical value. Therefore, further investigation should be addressed in order to explore the potential role of these promising markers.

\section{Competing Interests}

The authors declare that they have no competing interests.

\section{References}

[1] J. Ferlay, I. I. Soerjomataram, R. Dikshit et al., "Cancer incidence and mortality worldwide: sources, methods and major patterns in GLOBOCAN 2012," International Journal of Cancer, vol. 136, no. 5, pp. E359-E386, 2015.

[2] E. R. Fearon and B. Vogelstein, "A genetic model for colorectal tumorigenesis," Cell, vol. 61, no. 5, pp. 759-767, 1990.

[3] C. R. Boland, F. A. Sinicrope, D. E. Brenner, and J. M. Carethers, "Colorectal cancer prevention and treatment," Gastroenterology, vol. 118, no. 2, pp. S115-S128, 2000.

[4] D. J. Weisenberger, K. D. Siegmund, M. Campan et al., "CpG island methylator phenotype underlies sporadic microsatellite instability and is tightly associated with BRAF mutation in colorectal cancer," Nature Genetics, vol. 38, no. 7, pp. 787-793, 2006.

[5] B. Gustavsson, G. Carlsson, D. MacHover et al., "A review of the evolution of systemic chemotherapy in the management of colorectal cancer," Clinical Colorectal Cancer, vol. 14, no. 1, pp. $1-10,2015$.

[6] "NCCN Clinical Practice Guidelines in Oncology (NCCN Guidelines $^{5}$ ) Colon Cancer," Version 22016, 2015.

[7] T. André, C. Boni, M. Navarro et al., "Improved overall survival with oxaliplatin, fluorouracil, and leucovorin as adjuvant treatment in stage II or III colon cancer in the MOSAIC trial," Journal of Clinical Oncology, vol. 27, no. 19, pp. 3109-3116, 2009.

[8] H.-J. Schmoll, T. Cartwright, J. Tabernero et al., "Phase III trial of capecitabine plus oxaliplatin as adjuvant therapy for stage III colon cancer: a planned safety analysis in 1,864 patients," Journal of Clinical Oncology, vol. 25, no. 1, pp. 102-109, 2007.

[9] E. Van Cutsem, R. Labianca, G. Bodoky et al., "Randomized phase III trial comparing biweekly infusional fluorouracil/leucovorin alone or with irinotecan in the adjuvant treatment of stage III colon cancer: PETACC-3," Journal of Clinical Oncology, vol. 27, no. 19, pp. 3117-3125, 2009.

[10] S. Gill, C. L. Loprinzi, D. J. Sargent et al., "Pooled analysis of fluorouracil-based adjuvant therapy for stage II and III colon cancer: who benefits and by how much?" Journal of Clinical Oncology, vol. 22, no. 10, pp. 1797-1806, 2004.

[11] D. Ciliberto, U. Prati, L. Roveda et al., "Role of systemic chemotherapy in the management of resected or resectable colorectal liver metastases: a systematic review and meta-analysis of randomized controlled trials," Oncology Reports, vol. 27, no. 6, pp. 1849-1856, 2012.

[12] Z.-M. Wang, Y.-Y. Chen, F.-F. Chen, S.-Y. Wang, and B. Xiong, "Peri-operative chemotherapy for patients with resectable colorectal hepatic metastasis: a meta-analysis," European Journal of Surgical Oncology, vol. 41, no. 9, pp. 1197-1203, 2015.

[13] G. Colucci, V. Gebbia, G. Paoletti et al., "Phase III randomized trial of FOLFIRI versus FOLFOX4 in the treatment of advanced 
colorectal cancer: a Multicenter Study of the Gruppo Oncologico Dell'Italia Meridionale," Journal of Clinical Oncology, vol. 23, no. 22, pp. 4866-4875, 2005.

[14] J. Souglakos, N. Androulakis, K. Syrigos et al., "FOLFOXIRI (folinic acid, 5-fluorouracil, oxaliplatin and irinotecan) vs FOLFIRI (folinic acid, 5-fluorouracil and irinotecan) as firstline treatment in metastatic colorectal cancer (MCC): a multicentre randomised phase III trial from the Hellenic Oncology Research Group (HORG)," British Journal of Cancer, vol. 94, no. 6, pp. 798-805, 2006.

[15] A. Falcone, S. Ricci, I. Brunetti et al., "Phase III trial of infusional fluorouracil, leucovorin, oxaliplatin, and irinotecan (FOLFOXIRI) compared with infusional fluorouracil, leucovorin, and irinotecan (FOLFIRI) as first-line treatment for metastatic colorectal cancer: the Gruppo Oncologico Nor," Journal of Clinical Oncology, vol. 25, no. 13, pp. 1670-1676, 2007.

[16] J. Cassidy, S. Clarke, E. Díaz-Rubio et al., "Randomized phase III study of capecitabine plus oxaliplatin compared with fluorouracil/folinic acid plus oxaliplatin as first-line therapy for metastatic colorectal cancer," Journal of Clinical Oncology, vol. 26, no. 12, pp. 2006-2012, 2008.

[17] E. Van Cutsem, C.-H. Köhne, E. Hitre et al., "Cetuximab and chemotherapy as initial treatment for metastatic colorectal cancer," The New England Journal of Medicine, vol. 360, no. 14, pp. 1408-1417, 2009.

[18] H. Hurwitz, L. Fehrenbacher, W. Novotny et al., "Bevacizumab plus irinotecan, fluorouracil, and leucovorin for metastatic colorectal cancer," The New England Journal of Medicine, vol. 350, no. 23, pp. 2335-2342, 2004.

[19] U. McDermott, J. R. Downing, and M. R. Stratton, "Genomics and the continuum of cancer care," The New England Journal of Medicine, vol. 364, no. 4, pp. 340-350, 2011.

[20] A. Sobrero, A. Guglielmi, F. Grossi, F. Puglisi, and C. Aschele, "Mechanism of action of fluoropyrimidines: relevance to the new developments in colorectal cancer chemotherapy," Seminars in Oncology, vol. 27, no. 5, pp. 72-77, 2000.

[21] P. G. Johnston, H.-J. Lenz, C. G. Leichman et al., “Thymidylate synthase gene and protein expression correlate and are associated with response to 5-fluorouracil in human colorectal and gastric tumors," Cancer Research, vol. 55, no. 7, pp. 1407-1412, 1995.

[22] S. Popat, A. Matakidou, and R. S. Houlston, “Thymidylate-synthase expression and prognosis in colorectal cancer: a systematic review and meta-analysis," Journal of Clinical Oncology, vol. 22, no. 3, pp. 529-536, 2004.

[23] S. T. Pullarkat, J. Stoehlmacher, V. Ghaderi et al., “Thymidylate synthase gene polymorphism determines response and toxicity of 5-FU chemotherapy," Pharmacogenomics Journal, vol. 1, no. 1, pp. 65-70, 2001.

[24] K. Kawakami and G. Watanabe, "Identification and functional analysis of single nucleotide polymorphism in the tandem repeat sequence of thymidylate synthase gene," Cancer Research, vol. 63, no. 18, pp. 6004-6007, 2003.

[25] K. Uchida, K. Hayashi, K. Kawakami et al., "Loss of Heterozygosity at the Thymidylate Synthase (TS) locus on chromosome 18 affects tumor response and survival in individuals heterozygous for a 28-bp polymorphism in the TS gene," Clinical Cancer Research, vol. 10, no. 2, pp. 433-439, 2004.

[26] S. Tsujitani, H. Saito, Y. Maeta et al., "Neoangiogenesis in patients with gastric carcinoma in relation to the expression of vascular endothelial growth factor and thymidine phosphorylase," Anticancer Research, vol. 24, no. 3, pp. 1853-1859, 2004.
[27] E. H. Slager, M. W. Honders, E. D. van der Meijden et al., "Identification of the angiogenic endothelial-cell growth factor-1/ thymidine phosphorylase as a potential target for immunotherapy of cancer," Blood, vol. 107, no. 12, pp. 4954-4960, 2006.

[28] D.-J. Ye and J.-M. Zhang, "Research development of the relationship between thymidine phosphorylase expression and colorectal carcinoma," Cancer Biology \& Medicine, vol. 10, no. 1, pp. 10-15, 2013.

[29] H. Uetake, W. Ichikawa, T. Takechi, M. Fukushima, Z. Nihei, and K. Sugihara, "Relationship between intratumoral dihydropyrimidine dehydrogenase activity and gene expression in human colorectal cancer," Clinical Cancer Research, vol. 5, no. 10, pp. 2836-2839, 1999.

[30] M. Kornmann, W. Schwabe, S. Sander et al., "Thymidylate synthase and dihydropyrimidine dehydrogenase mRNA expression levels: predictors for survival in colorectal cancer patients receiving adjuvant 5-fluorouracil," Clinical Cancer Research, vol. 9, no. 11, pp. 4116-4124, 2003.

[31] D. Salonga, M. Johnson, R. Metzger et al., "Colorectal tumors responding to 5-fluorouracil have low gene expression levels of dihydropyrimidine dehydrogenase, thymidylate synthase, and thymidine phosphorylase," Clinical Cancer Research, vol. 6, no. 4, pp. 1322-1327, 2000.

[32] K. Omura, "Clinical implications of dihydropyrimidine dehydrogenase (DPD) activity in 5-FU-based chemotherapy: mutations in the DPD gene, and DPD inhibitory fluoropyrimidines," International Journal of Clinical Oncology, vol. 8, no. 3, pp. 132138, 2003.

[33] A. B. P. Van Kuilenburg, J. Haasjes, D. J. Richel et al., "Clinical implications of dihydropyrimidine dehydrogenase (DPD) deficiency in patients with severe 5 -fluorouracil-associated toxicity: identification of new mutations in the DPD gene," Clinical Cancer Research, vol. 6, no. 12, pp. 4705-4712, 2000.

[34] P. D. Senter, K. S. Beam, B. Mixan, and A. F. Wahl, "Identification and activities of human carboxylesterases for the activation of CPT-11, a clinically approved anticancer drug," Bioconjugate Chemistry, vol. 12, no. 6, pp. 1074-1080, 2001.

[35] L. Iyer, C. D. King, P. F. Whitington et al., "Genetic predisposition to the metabolism of irinotecan (CPT-11): role of uridine diphosphate glucuronosyltransferase isoform $1 \mathrm{~A} 1$ in the glucuronidation of its active metabolite (SN-38) in human liver microsomes," The Journal of Clinical Investigation, vol. 101, no. 4, pp. 847-854, 1998.

[36] F. Innocenti, S. D. Undevia, L. Iyer et al., "Genetic variants in the UDP-glucuronosyltransferase 1A1 gene predict the risk of severe neutropenia of irinotecan," Journal of Clinical Oncology, vol. 22, no. 8, pp. 1382-1388, 2004.

[37] X. Liu, D. Cheng, Q. Kuang, G. Liu, and W. Xu, “Association between $\mathrm{UGT1A1}^{\star} 28$ polymorphisms and clinical outcomes of irinotecan-based chemotherapies in colorectal cancer: a metaanalysis in caucasians," PLOS ONE, vol. 8, no. 3, article e58489, 2013.

[38] L. Iyer, S. Das, L. Janisch et al., "UGT1A1*28 polymorphism as a determinant of irinotecan disposition and toxicity," Pharmacogenomics Journal, vol. 2, no. 1, pp. 43-47, 2002.

[39] L. Cheng, M. Li, J. Hu et al., "UGT1A1*6 polymorphisms are correlated with irinotecan-induced toxicity: a system review and meta-analysis in Asians," Cancer Chemotherapy and Pharmacology, vol. 73, no. 3, pp. 551-560, 2014.

[40] Y. Shirota, J. Stoehlmacher, J. Brabender et al., "ERCC1 and thymidylate synthase mRNA levels predict survival for colorectal 
cancer patients receiving combination oxaliplatin and fluorouracil chemotherapy," Journal of Clinical Oncology, vol. 19, no. 23, pp. 4298-4304, 2001.

[41] M.-Y. Huang, H.-L. Tsai, C.-H. Lin et al., "Predictive value of ERCC1, ERCC2, and XRCC1 overexpression for stage III colorectal cancer patients receiving FOLFOX-4 adjuvant chemotherapy," Journal of Surgical Oncology, vol. 108, no. 7, pp. 457464, 2013.

[42] S. Li, L. Zhu, L. Yao, L. Xia, and L. Pan, "Association between ERCC1 and TS mRNA levels and disease free survival in colorectal cancer patients receiving oxaliplatin and fluorouracil (5-FU) adjuvant chemotherapy," BMC Gastroenterology, vol. 14, no. 1, article 154, 2014.

[43] C. Y. Kim, S. H. Seo, M. S. An et al., "ERCC1 as a predictive marker for FOLFOX chemotherapy in an adjuvant setting," Annals of Coloproctology, vol. 31, no. 3, pp. 92-97, 2015.

[44] M.-X. Li, X.-Y. Bi, H. Zhao et al., "Excision repair crosscomplementation group 1 is a prognostic biomarker in patients with colorectal cancer receiving chemotherapy," Chinese Medical Journal, vol. 129, no. 5, pp. 586-593, 2016.

[45] J. J. Yu, K. B. Lee, C. Mu et al., "Comparison of two human ovarian carcinoma cell lines (A2780/CP70 and MCAS) that are equally resistant to platinum, but differ at codon 118 of the ERCC1 gene," International Journal of Oncology, vol. 16, no. 3, pp. 555-560, 2000.

[46] A. Ruzzo, F. Graziano, F. Loupakis et al., "Pharmacogenetic profiling in patients with advanced colorectal cancer treated with first-line FOLFOX-4 chemotherapy," Journal of Clinical Oncology, vol. 25, no. 10, pp. 1247-1254, 2007.

[47] K. Sun, A. Gong, and P. Liang, "Predictive impact of genetic polymorphisms in DNA repair genes on susceptibility and therapeutic outcomes to colorectal cancer patients," Tumor Biology, vol. 36, no. 3, pp. 1549-1559, 2015.

[48] F. Ciardiello and G. Tortora, "EGFR antagonists in cancer treatment," The New England Journal of Medicine, vol. 358, no. 11, pp. 1160-1174, 2008.

[49] D. Cunningham, Y. Humblet, S. Siena et al., "Cetuximab monotherapy and cetuximab plus irinotecan in irinotecan-refractory metastatic colorectal cancer," The New England Journal of Medicine, vol. 351, no. 4, pp. 337-345, 2004.

[50] L. B. Saltz, N. J. Meropol, P. J. Loehrer Sr., M. N. Needle, J. Kopit, and R. J. Mayer, "Phase II trial of cetuximab in patients with refractory colorectal cancer that expresses the epidermal growth factor receptor," Journal of Clinical Oncology, vol. 22, no. 7, pp. 1201-1208, 2004.

[51] A. Lièvre, J.-B. Bachet, D. Le Corre et al., "KRAS mutation status is predictive of response to cetuximab therapy in colorectal cancer," Cancer Research, vol. 66, no. 8, pp. 3992-3995, 2006.

[52] F. Di Fiore, F. Blanchard, F. Charbonnier et al., "Clinical relevance of KRAS mutation detection in metastatic colorectal cancer treated by Cetuximab plus chemotherapy," British Journal of Cancer, vol. 96, no. 8, pp. 1166-1169, 2007.

[53] W. De Roock, H. Piessevaux, J. De Schutter et al., "KRAS wildtype state predicts survival and is associated to early radiological response in metastatic colorectal cancer treated with cetuximab," Annals of Oncology, vol. 19, no. 3, pp. 508-515, 2008.

[54] E. Van Cutsem, C.-H. Köhne, I. Láng et al., "Cetuximab plus irinotecan, fluorouracil, and leucovorin as first-line treatment for metastatic colorectal cancer: updated analysis of overall survival according to tumor KRAS and BRAF mutation status," Journal of Clinical Oncology, vol. 29, no. 15, pp. 2011-2019, 2011.
[55] E. Van Cutsem, H.-J. Lenz, C.-H. Köhne et al., "Fluorouracil, leucovorin, and irinotecan plus cetuximab treatment and RAS mutations in colorectal cancer," Journal of Clinical Oncology, vol. 33, no. 7, pp. 692-700, 2015.

[56] C. Bokemeyer, I. Bondarenko, J. T. Hartmann et al., "Efficacy according to biomarker status of cetuximab plus FOLFOX-4 as first-line treatment for metastatic colorectal cancer: the OPUS study," Annals of Oncology, vol. 22, no. 7, pp. 1535-1546, 2011.

[57] T. S. Maughan, R. A. Adams, C. G. Smith et al., "Addition of cetuximab to oxaliplatin-based first-line combination chemotherapy for treatment of advanced colorectal cancer: results of the randomised phase 3 MRC COIN trial," The Lancet, vol. 377, no. 9783, pp. 2103-2114, 2011.

[58] K. M. Tveit, T. Guren, B. Glimelius et al., "Phase III trial of cetuximab with continuous or intermittent fluorouracil, leucovorin, and oxaliplatin (Nordic FLOX) versus FLOX alone in firstline treatment of metastatic colorectal cancer: the NORDIC-VII study," Journal of Clinical Oncology, vol. 30, no. 15, pp. 1755-1762, 2012.

[59] C. J. Allegra, J. M. Jessup, M. R. Somerfield et al., "American Society of Clinical Oncology provisional clinical opinion: testing for KRAS gene mutations in patients with metastatic colorectal carcinoma to predict response to anti-epidermal growth factor receptor monoclonal antibody therapy," Journal of Clinical Oncology, vol. 27, no. 12, pp. 2091-2096, 2009.

[60] R. G. Amado, M. Wolf, M. Peeters et al., "Wild-type KRAS is required for panitumumab efficacy in patients with metastatic colorectal cancer," Journal of Clinical Oncology, vol. 26, no. 10, pp. 1626-1634, 2008.

[61] J.-Y. Douillard, S. Siena, J. Cassidy et al., "Randomized, Phase III trial of panitumumab with infusional fluorouracil, leucovorin, and oxaliplatin (FOLFOX4) Versus FOLFOX4 alone as first-line treatment in patients with previously untreated metastatic colorectal cancer: The PRIME study," Journal of Clinical Oncology, vol. 28, no. 31, pp. 4697-4705, 2010.

[62] F. Di Nicolantonio, M. Martini, F. Molinari et al., "Wild-type $\mathrm{BRAF}$ is required for response to panitumumab or cetuximab in metastatic colorectal cancer," Journal of Clinical Oncology, vol. 26, no. 35, pp. 5705-5712, 2008.

[63] M. Frattini, D. Balestra, S. Suardi et al., "Different genetic features associated with colon and rectal carcinogenesis," Clinical Cancer Research, vol. 10, no. 12, pp. 4015-4021, 2004.

[64] H. Rajagopalan, A. Bardelli, C. Lengauer, K. W. Kinzler, B. Vogelstein, and V. E. Velculescu, "Tumorigenesis: RAF/RAS oncogenes and mismatch-repair status," Nature, vol. 418, no. 6901, article 934, 2002.

[65] W. De Roock, B. Claes, D. Bernasconi et al., "Effects of KRAS, BRAF, NRAS, and PIK3CA mutations on the efficacy of cetuximab plus chemotherapy in chemotherapy-refractory metastatic colorectal cancer: a retrospective consortium analysis," The Lancet Oncology, vol. 11, no. 8, pp. 753-762, 2010.

[66] C. Bokemeyer, E. V. Cutsem, P. Rougier et al., "Addition of cetuximab to chemotherapy as first-line treatment for KRAS wild-type metastatic colorectal cancer: pooled analysis of the CRYSTAL and OPUS randomised clinical trials," European Journal of Cancer, vol. 48, no. 10, pp. 1466-1475, 2012.

[67] J.-Y. Douillard, K. S. Oliner, S. Siena et al., "PanitumumabFOLFOX4 treatment and RAS mutations in colorectal cancer," The New England Journal of Medicine, vol. 369, no. 11, pp. 10231034, 2013.

[68] M. Frattini, P. Saletti, E. Romagnani et al., "PTEN loss of expression predicts cetuximab efficacy in metastatic colorectal 
cancer patients," British Journal of Cancer, vol. 97, no. 8, pp. 1139$1145,2007$.

[69] A. Sartore-Bianchi, M. Martini, F. Molinari et al., "PIK3CA mutations in colorectal cancer are associated with clinical resistance to EGFR-targeted monoclonal antibodies," Cancer Research, vol. 69, no. 5, pp. 1851-1857, 2009.

[70] F. Perrone, A. Lampis, M. Orsenigo et al., "PI3KCA/PTEN deregulation contributes to impaired responses to cetuximab in metastatic colorectal cancer patients," Annals of Oncology, vol. 20, no. 1, pp. 84-90, 2009.

[71] C. S. Karapetis, D. Jonker, M. Daneshmand et al., "PIK3CA, BRAF, and PTEN status and benefit from cetuximab in the treatment of advanced colorectal cancer-results from NCIC CTG/AGITG CO.17," Clinical Cancer Research, vol. 20, no. 3, pp. 744-753, 2014.

[72] R. Avraham and Y. Yarden, "Feedback regulation of EGFR signalling: decision making by early and delayed loops," Nature Reviews Molecular Cell Biology, vol. 12, no. 2, pp. 104-117, 2011.

[73] B. Jacobs, W. De Roock, H. Piessevaux et al., "Amphiregulin and epiregulin mRNA expression in primary tumors predicts outcome in metastatic colorectal cancer treated with cetuximab," Journal of Clinical Oncology, vol. 27, no. 30, pp. 5068-5074, 2009.

[74] S. Khambata-Ford, C. R. Garrett, N. J. Meropol et al., "Expression of epiregulin and amphiregulin and K-ras mutation status predict disease control in metastatic colorectal cancer patients treated with cetuximab," Journal of Clinical Oncology, vol. 25, no. 22, pp. 3230-3237, 2007.

[75] M. V. Blagosklonny, "How avastin potentiates chemotherapeutic drugs: action and reaction in antiangiogenic therapy," Cancer Biology \& Therapy, vol. 4, no. 12, pp. 1307-1310, 2005.

[76] P. S. Hegde, A. M. Jubb, D. Chen et al., "Predictive impact of circulating vascular endothelial growth factor in four phase III trials evaluating bevacizumab," Clinical Cancer Research, vol. 19, no. 4, pp. 929-937, 2013.

[77] H. Hayashi, T. Arao, K. Matsumoto et al., "Biomarkers of reactive resistance and early disease progression during chemotherapy plus bevacizumab treatment for colorectal carcinoma," Oncotarget, vol. 5, no. 9, pp. 2588-2595, 2014.

[78] J. M. Jürgensmeier, H.-J. Schmoll, J. D. Robertson et al., "Prognostic and predictive value of VEGF, sVEGFR-2 and CEA in mCRC studies comparing cediranib, bevacizumab and chemotherapy," British Journal of Cancer, vol. 108, no. 6, pp. 1316-1323, 2013.

[79] M. A. Bruhn, A. R. Townsend, C. Khoon Lee et al., "Proangiogenic tumor proteins as potential predictive or prognostic biomarkers for bevacizumab therapy in metastatic colorectal cancer," International Journal of Cancer, vol. 135, no. 3, pp. 731741, 2014.

[80] H.-L. Tsai, C.-H. Lin, C.-W. Huang et al., "Decreased peritherapeutic VEGF expression could be a predictor of responsiveness to first-line FOLFIRI plus bevacizumab in mCRC patients," International Journal of Clinical and Experimental Pathology, vol. 8, no. 2, pp. 1900-1910, 2015.

[81] F. Loupakis, C. Cremolini, A. Fioravanti et al., "Pharmacodynamic and pharmacogenetic angiogenesis-related markers of first-line FOLFOXIRI plus bevacizumab schedule in metastatic colorectal cancer," British Journal of Cancer, vol. 104, no. 8, pp. 1262-1269, 2011.

[82] C. Fischer, B. Jonckx, M. Mazzone et al., "Anti-PlGF Inhibits Growth of VEGF(R)-inhibitor-resistant tumors without affecting healthy vessels," Cell, vol. 131, no. 3, pp. 463-475, 2007.
[83] A. Pohl, A. El-Khoueiry, D. Yang et al., "Pharmacogenetic profiling of CD133 is associated with response rate (RR) and progression-free survival (PFS) in patients with metastatic colorectal cancer (mCRC), treated with bevacizumab-based chemotherapy," Pharmacogenomics Journal, vol. 13, no. 2, pp. 173-180, 2013.

[84] T. Purnak, E. Ozaslan, and C. Efe, "Molecular basis of colorectal cancer," The New England Journal of Medicine, vol. 362, no. 13, pp. 1246-1247, 2010.

[85] M. Koopman, G. A. M. Kortman, L. Mekenkamp et al., "Deficient mismatch repair system in patients with sporadic advanced colorectal cancer," British Journal of Cancer, vol. 100, no. 2, pp. 266-273, 2009.

[86] C. M. Ribic, D. J. Sargent, M. J. Moore et al., “Tumor microsatellite-instability status as a predictor of benefit from fluorouracil-based adjuvant chemotherapy for colon cancer," The New England Journal of Medicine, vol. 349, no. 3, pp. 247-257, 2003.

[87] D. J. Sargent, S. Marsoni, G. Monges et al., "Defective mismatch repair as a predictive marker for lack of efficacy of fluorouracilbased adjuvant therapy in colon cancer," Journal of Clinical Oncology, vol. 28, no. 20, pp. 3219-3226, 2010.

[88] A. D. Roth, S. Tejpar, M. Delorenzi et al., "Prognostic role of KRAS and BRAF in stage II and III resected colon cancer: results of the translational study on the PETACC-3, EORTC 40993, SAKK 60-00 trial," Journal of Clinical Oncology, vol. 28, no. 3, pp. 466-474, 2010.

[89] J. F. Flejou, T. André, B. Chibaudel et al., "Effect of adding oxaliplatin to adjuvant 5 -fluorouracil/leucovorin (5FU/LV) in patients with defective mismatch repair (dMMR) colon cancer stage II and III included in the MOSAIC study," Journal of Clinical Oncology, vol. 31, supplement, abstract 3524, 2013.

[90] E. M. Webber, T. L. Kauffman, E. O'Connor, and K. Goddard, "Systematic review of the predictive effect of MSI status in colorectal cancer patients undergoing 5FU-based chemotherapy," BMC Cancer, vol. 15, no. 1, article 156, 2015. 


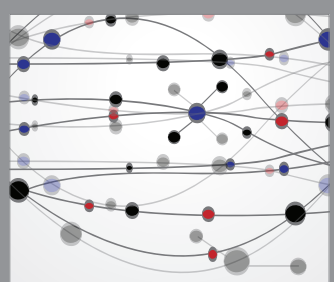

The Scientific World Journal
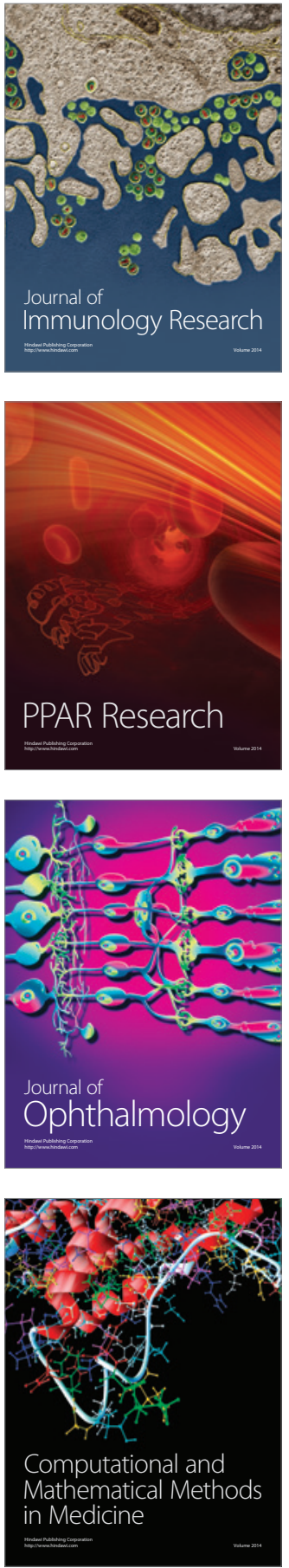

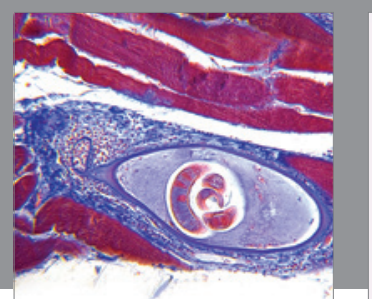

Gastroenterology Research and Practice

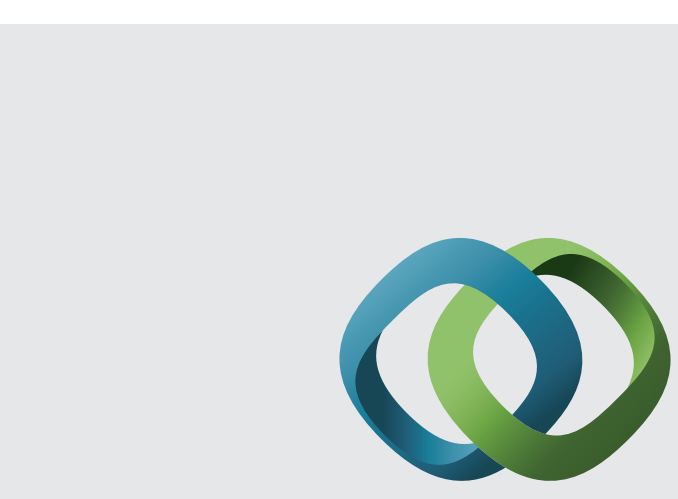

\section{Hindawi}

Submit your manuscripts at

http://www.hindawi.com
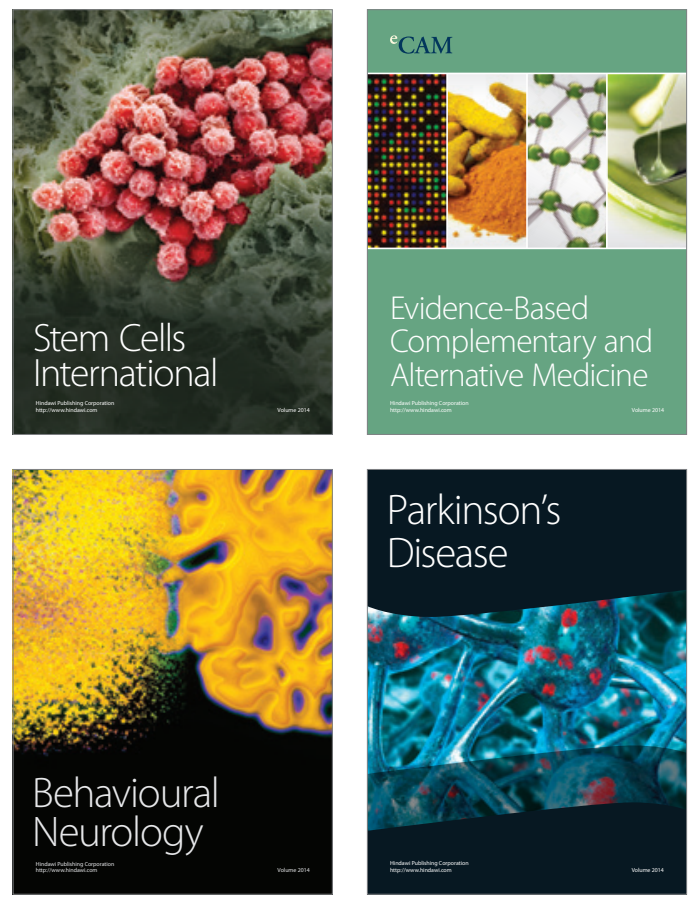
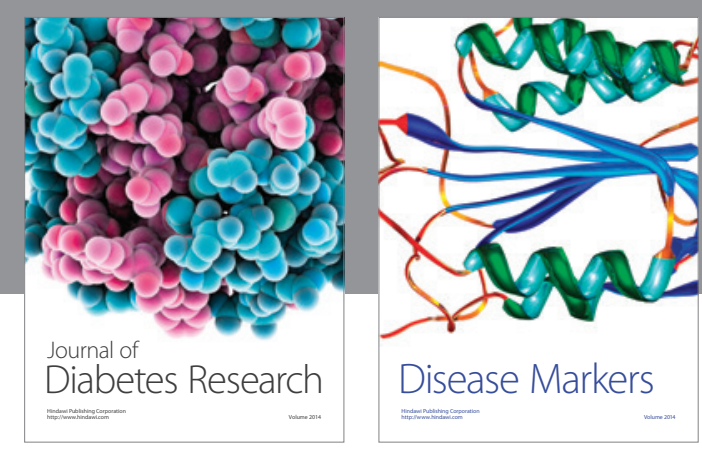

Disease Markers
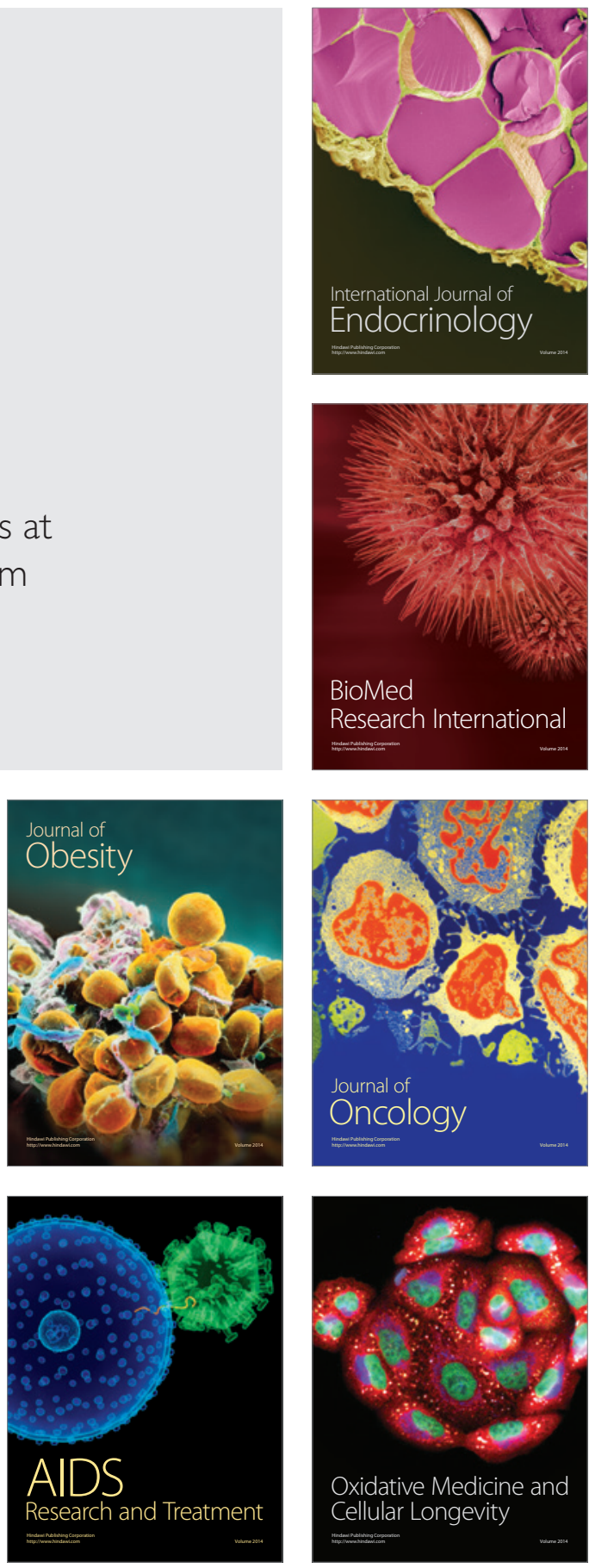